\title{
ANNA SZEMBERSKA
}

Università Adam Mickiewicz, Poznań

\section{LINGUA DEL CALCIO COME UNA LINGUA SETTORIALE}

\begin{abstract}
A bstract. Szemberska Anna, Lingua del calcio come una lingua settoriale [The Italian of football as a special language], Studia Romanica Posnaniensia, Adam Mickiewicz University Press, Poznań, vol. XXXVII/2: 2010, pp. 95-106. ISBN 978-83-232-2189-0. ISSN 0137-2475. DOI 10.2478/v10123-0100018-y.
\end{abstract}

The main motives of this article are the tecnical terms used by journalists in the Italian football press. The paper presents the Italian of football, its multiple aspects and its characteristics as a special language. The aim of this article, in which we also consider the popularity of football press in Italy, is to analyse its semantic, stilistic, syntactic and textual features.

\section{INTRODUZIONE}

Il presente saggio ha per oggetto l'analisi del linguaggio del calcio delle cronache sportive in quanto una lingua settoriale. Tra le varie lingue settoriali quella del calcio, un po' sottovalutata ma ciononostante feconda e ricca di varie espressioni, neologismi e prestiti, costituisce una fonte quasi inesauribile e un campo di ricerca alquanto promettente. Va preso in considerazione che tanti modi di dire entrati nell'intercalare di tutti i giorni hanno le loro origini proprio nel lessico calcistico o quantomeno sportivo. Il linguaggio del calcio è un settore della lingua che subisce una metamorfosi continua, in quanto la lingua stessa è un organismo dinamico che si arricchisce ed entra in comunicazione con altri ambiti; questo spiega anche la presenza di tante espressioni sportive nella lingua di tutti i giorni, ad esempio fair play, fare gol (centrare, azzeccare), dare il fischio d'inizio (segno di cominciare) o fare squadra (agire con lo spirito di gruppo). La prova della presenza del linguaggio del calcio nella vita quotidiana degli italiani sono le seguenti citazioni dai giornali; una usata per commentare la recente situazione di politica interna: „Ma spunta il terzo uomo pressing su Chiamparino"' e l'altra - riguardante la situazione nel Partito Democratico: „Pd, tessere in zona Cesarini"'?.

\footnotetext{
${ }^{1} \mathrm{http}: / /$ www.repubblica.it/, 26-6-2009.

${ }^{2} \mathrm{http}: / /$ www.repubblica.it/, 19-7-2009. Con questo termine vengono designati infatti gli ultimi minuti di una partita di calcio (in particolar modo nell'espressione segnare in zona Cesarini). La locuzione, propagata dai giornalisti sportivi, è diventata proverbiale ed è riuscita ad imporsi anche nel lessico dei commentatori politici e nell'italiano di uso comune. Attualmente zona Cesarini è una
} 
La scelta della materia d'indagine è stata suggerita dalla scarsezza di studi in merito: infatti, le ricerche specifiche sull'argomento si riducono a pochi brevi contributi apparsi tra l'altro sulla rivista Lingua Nostra. Un ulteriore spunto per trattare quest'argomento, linguisticamente molto avvincente, ci è stato offerto dall'articolo di Giacomo Devoto, pubblicato nel 1939 sulla prestigiosa rivista fiorentina, in cui l'illustre italianista scrisse che:

[...] la storia del lessico calcistico italiano è un argomento più interessante e fruttuoso come tesi di laurea in lettere di tanti altri più o meno illustri e rifritti, importa ora stabilire che il lessico, per quanto importante in sé, è soltanto un mezzo di definizione di una lingua speciale: esso si inserisce, senza possibilità di confini netti, nell'insieme degli effetti e dei contrasti in una lingua ${ }^{3}$.

\section{GIORNALISMO SPORTIVO IN ITALIA}

A Devoto fa verso anche Vittorio Roidi affermando che „Lo sport è stato un buon volano per il giornalismo. Protagonista assoluto è il calcio, con i suoi eccessi e le sue storture, fenomeno socio-culturale a più facce che ha grande influenza sulla formazione dei giovani e sul modo di trascorrere il tempo libero. Lo sport vive di informazione, visto che sono più numerosi coloro che ne parlano, rispetto a coloro che lo praticano"'. Per tale motivo la fonte principale di quest'articolo, oltre alle pubblicazioni riguardanti il calcio, è costituita dalla stampa sportiva quotidiana, nella quale a innumerevoli incontri calcistici spesso corrispondono altrettante parole nuove, inventate da cronisti, telecronisti e giocatori. Come osservano Della Valle e Adamo, non di rado si tratta di formazioni legate a episodi che colpiscono l'immaginazione collettiva, grazie anche all'innegabile fantasia degli stessi giornalisti contribuenti alla creazione e alla diffusione di esse $^{5}$. Va però sottolineato che alcune di queste neoformazioni riescono ad incontrare un favore e un successo immediati, mentre altre si rivelano del tutto effimere. Inoltre, come afferma Roidi: „Secondo i ricercatori dell'Eurispes e del Censis solo 14 studenti su cento leggono ogni giorno un giornale e chi lo fa gli dedica appena una decina di minuti. Il $26 \%$ rivolge l'attenzione ai titoli sportivi, il $20 \%$ alla cronaca e solo il $7 \%$ alla politica. Il $23 \%$ legge l'oroscopo. I ragazzi non hanno dimestichezza con il quotidiano, non vi trovano cose a loro parere interessanti. Cambiamo i ragazzi o i giornali?"'.

Sono sufficienti queste due considerazioni pungenti di Roidi per capire l'importanza che la stampa sportiva ricopre nel panorama del giornalismo in Italia. I pochi

delle antonomasie più diffuse della lingua italiana con il significato all'ultimo minuto. Come sinonimo appare anche il latinismo in extremis.

${ }^{3}$ Lingua Nostra, settembre-dicembre 1939, p. 11.

${ }^{4}$ Roidi (2001, p. 189).

5 Cfr. Adamo, Della Valle (2005, p. VI).

${ }^{6}$ Roidi (2001, p. 10). 
lettori di quotidiani dedicano grande interesse alle pagine sportive, perciò chi in Italia legge un giornale, nella maggior parte dei casi legge anche (o soprattutto) le cronache calcistiche. Basta scorrere i dati Audipress risalenti al gennaio 2008 che indicano $\mathrm{La}$ Gazzetta dello Sport come il quotidiano italiano più letto in assoluto. A seguire ci sono La Repubblica, il Corriere della Sera e La Stampa. Il quarto posto in assoluto occupa il Corriere dello Sport-Stadio. La centralità dello sport nell'ambito dell'informazione italiana pare dunque evidente se consideriamo che, oltre al crescente numero delle pagine sportive nei quotidiani, in Italia esistono ormai dal 1945 - anno in cui Renato Casalbore fondò a Torino Tuttosport che si andava ad aggiungere alla meneghina Gazzetta dello Sport (1896) e al romano Corriere dello Sport (1942) - ben tre quotidiani dedicati allo sport (caso unico in Europa), acquistati regolarmente da 800000 lettori. Se si pensa che in totale $i$ consumatori abituali di stampa quotidiana sono poco più di 6 milioni, risulta lampante che i lettori „sportivi” costituiscono una fetta decisamente rilevante del mercato giornalistico italiano ${ }^{7}$.

Nel presente articolo, quindi, si toccheranno gli argomenti riguardanti la lingua del calcio come sottocodice ${ }^{8}$.

\section{LINGUA DEL CALCIO COME LINGUA SETTORIALE}

La lingua del calcio in quanto lingua settoriale rappresenta una varietà della lingua naturale, dipendente da un settore di conoscenze o da un ambito di attività professionali. Una lingua settoriale è utilizzata da un gruppo di parlanti più ristretto rispetto a quelli che parlano la lingua base e risponde all'esigenza di un certo settore specialistico, nel nostro caso quello sportivo. Secondo le considerazioni di Serianni „In molti casi [...] i linguaggi settoriali ricorrono al meccanismo della rideterminazione, cioè assegnano un significato specifico a parole d'uso comune, generando possibili equivoci" $"$. Per dare un esempio, nel lessico calcistico curva sta per indicare in uno stadio l'insieme dei posti collocati dietro le porte e la tifoseria che abitualmente vi prende posto: curva Nord, curva Sud. Ma curva è anche un termine del linguaggio comune abitualmente usato per indicare parte di pista atletica o di percorso che disegna un arco. Come altro esempio possiamo citare il termine finta che nel linguaggio militare indica un'azione simulata per distrarre il nemico dal punto in cui lo si vuole attaccare. Per estensione, questa locuzione viene usata nel calcio con il significato di movimento accennato ma non portato a termine, per ingannare l'avversario: fare una finta, una serie di finte. Comunemente la parola catenaccio designa una sbarra di ferro che scorre in anelli infitti nei battenti di porte e sim. per chiuderli. Nel senso calcistico

\footnotetext{
${ }^{7}$ Fonte dati: www.audipress.it; i dati del 2008/I.

${ }^{8}$ Quelle che per Sobrero sono le „lingue settoriali”, da Berruto vengono definite ,sottocodici” e da Bonomi (2002, p. 184) invece „linguaggi speciali”; cfr. Sobrero (1993, pp. 238-239), Berruto (1976, pp. 68-73).

${ }^{9}$ Serianni (2003, p. 80).
} 
catenaccio vuol dire tattica rigidamente difensiva: una squadra ha fatto del catenaccio; si usa anche l'aggettivo derivato da questo termine: squadra catenacciara, calcio catenacciaro. Consideriamo un altro esempio: nell'architettura il termine lunetta è un elemento di muratura, a forma semicircolare, che sovrasta una porta o una finestra. Nel linguaggio del calcio invece esso indica lo spazio di campo compreso tra il semicerchio disegnato dall'area di rigore in corrispondenza della porta e al limite dell'area di rigore stessa. Gli esempi citati dimostrano che questi termini, entrando nella lingua del calcio, hanno subito un' evoluzione a partire dal significato di base. È anche il caso del verbo fulminare usato nello sport nel significato di sorprendere il portiere con un tiro imprendibile.

Bonomi (2002, p. 184) invece sotto termine ,voci di linguaggi speciali” ragruppa sia i termini la cui diffusione si può circoscrivere a zone linguistiche settoriali sensu stricte, sia quelli ,usciti dall'ambito del linguaggio speciale cui appartenevano originariamente per divenire patrimonio di cerchie più o meno ampie di parlanti". Di conseguenza la studiosa fa notare (Bonomi, 2002, pp. 184-185) che bisogna inoltre distinguere due categorie di voci che del tutto differiscono tra loro. Si tratta di tecnicismi usati in senso proprio, molti dei quali hanno ,una diffusione estremamente circoscritta, limitata al linguaggio settoriale”, e di ,,voci tecniche impiegate in senso metaforico [...]". Una tale tendenza al trapasso dal tecnico al metaforico, come ricorda la linguista (Bonomi, 2002, pp. 184-185), „oggi particolarmente viva, agiva anche nell'italiano ottocentesco, anche se con forza decisamente minore rispetto alla lingua odierna, ed era da più parti combattuta, soprattutto in considerazione degli effetti di scarsa chiarezza e di affettazione che poteva provocare nel contesto".

A Bonomi fa eco Sobrero (1993, p. 240) secondo il quale le lingue specialistiche „hanno una zona di sovrapposizione con la lingua comune [...]”. La portata di essa varierebbe non in ragione dei singoli settori, ma dipenderebbe più che altro da competenze specifiche del singolo parlante. Lo studioso per esemplificare la sua teoria riporta le seguenti parole ed espressioni tecniche: smarcare, crossare, penalty, zona, quattro due quattro, pressing. Esse, stando la linguista, non si possono classificare come ,intrinseche alla lingua comune” oppure solamente ad essa ,tangenti”: secondo Sobrero (1993, p. 240) sarebbero classificabili „esclusivamente in riferimento al contesto in cui compaiono, e soprattutto all'interesse e alle esperienze degli interlocutori che, di volta in volta, le usano o le ascoltano".

Tornando alle caratteristiche di un sottocodice, un lessico specifico si distingue anche per la sua referenzialità e per la precisione. E così sono chiaramente identificati i termini come zona (impostazione tattica, distinta da quella ,a uomo", basata sulla copertura, da parte dei giocatori, di una determinata area del campo), muro, cucchiaio (tiro dalla parabola alta che scende all'improvviso, in modo da superare il portiere avversario) o poker (quattro gol segnati da una squadra).

A livello linguistico un sottocodice si caratterizza in primo luogo per determinate scelte lessicali ma anche morfologiche e sintattiche. A distinguerlo sono soprattutto 
i tecnicismi specifici ${ }^{10}$ però come abbiamo visto prima, in molti casi anche le parole dalla lingua comune assumono un significato specialistico attraverso fasi successive di impieghi metaforici o di ridefinizioni e vengono definite tecnicismi collaterali ${ }^{11}$ : rigore, cucchiaio, dischetto (circolo su cui si colloca la palla per l'effettuazione del calcio di rigore, detto anche tiro dal dischetto), rosa (insieme dei calciatori a disposizione di un allenatore), bidone (giocatore di scarsa abilità che si rivela inferiore alle aspettative, soprattutto in quanto ingaggiato per una cifra molto alta), rosso / giallo (cartellino), bandiera (giocatore che da anni milita nella stessa squadra) che esistono nella lingua comune ma con un altro significato. Il contributo dei tecnicismi collaterali è rendere un argomento più elevato, come si vuole dal seguente esempio:

In avvio di ripresa le cose non cambiano: il Benfica va vicino al gol al 3' con un colpo di testa di Saviola a lato. Borriello e Pato si trovano al 10': cross del primo, testa del secondo, palla alta. Tre minuti dopo il Benfica passa in vantaggio: bel traversone di Shaffer, Cardozo in area sorprende i centrali del Milan e di testa batte Storari. Poi Jorge Jesus sostituisce quasi tutti i suoi, la reazione del Milan si riassume in una conclusione rasoterra di Borriello, parata da Moreira. Neanche Ronaldinho, entrato per Di Gennaro poco prima del gol portoghese, riesce a dare la scossa. Quando il risultato sembra scritto, all'86' Pato si invola sulla sinistra, supera un difensore e mette al centro, dove Sidnei anticipa l'intervento di Borriello e batte il proprio portiere riportando il match in parità. In pieno recupero è Borriello ad andare vicino al gol con un gran sinistro dal limite. Si va ai rigori: dal dischetto segnano Pirlo, Jankulovski e Borriello, sbagliano Ronaldinho, Thiago Silva e Zambrotta; poi è l'errore decisivo di Antonini a regalare il trofeo al Benfica ${ }^{12}$.

I termini come cross (traversone), traversone, (tiro) rasoterra, parata, fanno parte di tecnicismi specifici, mentre area (ciascuna delle due zone prospicienti le porte, in cui i falli dei difensori sono puniti con il calcio di rigore), ripresa, testa sono le parole della lingua comune, risemantizzate.

\subsection{TRATTI STILISTICO-TESTUALI}

Il tratto stilistico-testuale più significativo del linguaggio calcistico è costituito dall'uso eccezionalmente ampio di figure retoriche. Questo ricorso alla sfera dell'immaginazione è legato all'esigenza di catturare l'attenzione del lettore tramite scelte linguistiche accattivanti. Fin dall'antichità la tradizione retorica in modo felicemente contraddittorio ,definisce la figure come modi di parlare lontani da quelli che sono naturali e ordinari o anche [...] semplici e comuni; ma nello stesso tempo nulla è più comune e ordinario dell'uso delle figure e si fanno molte più figure in un giorno di mercato in piazza di quante non se ne facciano in più giorni di assemblee accademiche"13.

\footnotetext{
${ }^{10}$ Parole usate nelle loro accezioni tecniche, indispensabili alle esigenze terminologiche di un linguaggio settoriale; cfr. Serianni (2003, p. 82).

${ }^{11} \mathrm{Al}$ contrario degli specifici, sostituibili senza che l'esattezza ne risenta.

${ }^{12}$ www.gazzetta.it, 8-8-2009.

${ }^{13}$ Genette (1969, pp. 190-191).
} 
Come sostiene Genette, ,la figura rappresenta una deviazione in rapporto all'uso comune, la deviazione è tuttavia nell'uso comune: ecco il paradosso della retorica" ${ }^{\prime 14}$. $\mathrm{Ne}$ risulta la constatazione che il linguaggio del calcio nel ricorso all'iperbole, con la sua affettazione e la ricercatezza, può essere altrettanto semplice. L'uso delle metafore e l'allargamento del significato servono per rendere enfatico un articolo sportivo e per questo esigono la fantasia dello scrivente:

I Faraoni (giocatori delle squadra dell'Egitto) si abbracciano tutti insieme, formando una piramide che stavolta ha sapore di vita e non di morte. Non ci credono neppure loro di aver battuto i campioni del Mondo (gazzetta.it, 19-6-09);

Non è un caso che il gol che cambia l'inerzia del match arrivi proprio grazie a una „trivela" del portoghese che incoccia la coscia di Grygera e smarca in modo decisamente fortunoso Thiago Motta. Quest'ultimo, per nulla imbarazzato dal gentile cadeau, con un „cucchiaino” batte Buffon (Ts, 15-8-09, p. 2);

Ancora pari, trazione amichevole tra Olanda e Inghilterra degli ultimi 4 confronti. Fabio Capello riporta in rotta i Leoni, perduti e battuti nel primo tempo. Intervento da guru nell'intervallo, con le sostituzioni che cambiano subito il match alla ripresa. Il disastro annunciato si trasforma in riscatto promettente. Dopo lo show oranje, Capello mette in vetrina le seconde scelte, nell'occasione meglio dei titolari consolidati. A cominciare da Defoe, due gol in risposta alle fiammate di Kuyt e Van der Vaart. «Non posso clonare Steve», aveva detto alla vigilia il c.t. inglese. Finora lo aveva sempre chiamato Gerrard, come gli altri, Lampard, Terry, da preside all'antica. «Steve» è tornato a Liverpool a risparmiare gli adduttori per il via della Premier. Il film della gara Debutto titolare per Ashley Young, che è «young», 24 anni, dell'Aston Villa. «Sa dribblare», scrive in pagella Capello. Ma non è Steve. E l'Olanda la fa da padrona, nel primo tempo. Non solo per l'insostenibile leggerezza di Young. I guai inglesi sono i buchi dietro. La giostra olandese, che parte in allegria, caglia la linea bianco latte davanti a Green (portiere di terza scelta), con un effetto Leerdammer. Errori individuali stroncano i tentativi di resistenza inglese di fronte alle creazioni di Van der Vaart (preferito a Sneijder, e se lo merita), al brio di Robben, alle stoccate-lampo di Kuyt, minaccia costante. Così Rio Ferdinand va in blackout appena dopo 10': offre la palla allo scatto di Kuyt, che danza attorno a Green e spara in gol (gazzetta.it, 13-8-09);

Sembrava avere 3 gambe, l'8 del Madrid, perché i suoi rivali, che ne hanno due, lo vedevano passare come un bolide sulla strada verso la porta. Ogni sua apparizione supera la precedente" (gazzetta.it, 16-8-09); „Kakà illumina un Madrid in costruzione” (gazzetta.it, 16-8-09); ,Imperiale nella conduzione della palla, millimetrico nel passaggio, violento nel tiro, il busto eretto, la testa alta. Il brasiliano con la sua onnipresenza ha contenuto da solo il coraggioso inizio della squadra di casa (gazzetta it. 16-8-09).

Tra le figure di parola più frequenti notiamo la metonimia: „Helveg: «Attenti, abbiamo la panchina lunga»" (gazzetta.it, 10-6-09) - i giocatori di riserva che durante la partita sono seduti in panchina; e l'antonomasia, adoperata spesso con finalità apologetiche per attribuire un nome di personaggio famoso a chi ne possiede le caratteristiche personali (come ad esempio Pinturicchio ${ }^{15}$ per Alessandro Del Piero:

${ }^{14}$ Genette (1969, pp. 190-191).

${ }^{15}$ Così lo battezzò Gianni Agnelli: „Sei un giovane pittore poco conosciuto, impegnati a fare capolavori degni di Raffaello"; con il riferimento ai colori della casacca della squadra del Siena - 
„Entusiasmo. Tutto esaurito davanti al «Summer Village» per le prime parole di Pinturicchio" [gazzetta.it, 13-7-09]).

Si può inoltre sostituire un nome comune o una perifrasi ad un nome proprio per personaggi celebri, o viceversa.

Con questo procedimento si creano i nomi e i soprannomi o si riassumono sinteticamente intere categorie così che nascono dei nuovi nomi comuni. Ad esempio: Avvocato al posto di Gianni Agnelli oppure el pibe de oro ${ }^{16}$ al posto di Maradona.

Frequentemente nell'introduzione ad un articolo troviamo una serie di ripetizioni, si veda l'esempio seguente:

Cambia la città, non il risultato finale. Cambia latitudine e continente, però il Milan soccombe sempre nei confronti estivi contro l'Inter (Ts, 15-8-09, p. 2).

Nella stessa maniera comincia un altro articolo sullo stesso quotidiano:

Attenti ai segnali (tit.) /Attenzione ai segnali. Il primo Ferrara-Mourinho 1-0. [...]. Secondo segnale: a otto giorni dall'inizio del campionato e a meno di quattro mesi dalla vera Juventus-Inter, la squadra di Ciro Ferrara è parsa ancora un po' troppo impacciata. [...]. Terzo segnale: Amauri è in forma strepitosa e, con i gol di ieri, ha completato un'estate brillante. Quarto segnale: il Milan ha mostrato molti dei vecchi limiti e pochissime felici novità [...] (Ts, 15-8-09, p. 1);

ed anche:

(Amauri) Segna a raffica perché per un attaccante il gol è la linfa vitale, segna tanto perché vuole dimostrare che il suo posto è alla Juventus. Segna da bomber di razza, approfittando dell'errore di Nelson Rivas, con un destro in diagonale su cui Julio Cesar è costretto ad arrendersi e si ripete nel match con il Milan beffando Flavio (Ts, 15-8-09, p. 3).

Le ripetizioni e le frasi nominali vengono usate per marcare emotivamente un dato evento calcistico.

Tra le diverse figure che caratterizzano le cronache delle partite di calcio, oltre a quelle già citate, troveremo l'antitesi: „Rischia di essere uno di troppo, ma quando gioca non è mai uno di meno". Un altro procedimento frequente è l'uso delle citazioni che molto spesso costituiscono interi titoli, in modo da agevolare la decontestualizzazione. Lontano dal contesto dell'articolo o della frase in cui vengono pronunciate, rischiano di apparire controverse o quantomeno discutibili e sono un modo furbo di attirare l'attenzione del lettore. Così fu dopo l'eliminazione degli Azzurri dal Mondiale nippocoreano nel 2002 - dalla prima pagina del Corriere dello Sport un enorme titolone a caratteri cubitali gridava: „LADRI!!"17.Tra le altre figure che servono per marcare intensivamente un articolo segnaliamo l'imprescindibile presenza dei polittoti $^{18}$ come nel frammento che segue: „Per una volta non ci sono freni a condizionare bianconeri, come quegli juventini; http://www.gazzetta.it/, 13-1-2006.

16 Spagn. ragazzo d'oro.

${ }^{17}$ Frase ripetuta dai giocatori e dirigenti azzurri dopo una serie di arbitraggi scandalosi; Corriere dello Sport, 19-6-2002, p. 1.

${ }^{18}$ Figura di ripetizione che consiste nella ricorrenza di una parola con funzioni sintattiche differenti, sia nello stesso enunciato, sia in quelli vicini; cfr. Moretti (1996, p. 674). 
la grandezza dell'Inter. Solo i freni del contegno, della serietà di fondo, a condizionare le parole del tecnico argentino"19. Anch'essi appaiono per sottolineare un'impresa sportiva, come altri procedimenti aventi lo scopo di mettere in particolare rilievo una data notizia, tra i quali l'iperbato ${ }^{20}$ : „Continuerà Francesco Toldo, uno che all'Arsenal, nella sua carriera, ha sempre fatto male"21. Il rovesciamento dell'ordine abituale di alcuni elementi della frase consiste in questo caso nell'inserire il complemento di luogo nella sua carriera prima del sintagma verbale. Sono anche frequenti le similitudini („La partenza è come da copione”; gazzetta.it, 19-8-09) e i polisindeti22: „Il passaggio dell'ex granata, (e interista, e parmigiano) Hakan Sukur l'ha narcotizzata"23. Alla sinteticità del linguaggio della stampa si combina bene l'uso di elissi: il destro / il sinistro (tiro effettuato con il piede destro / sinistro), od anche il seguente esempio:

Confortante invece la prova di Bogliacino, le progressioni di Hamsik e la duttilità di Campagnaro, efficace nelle sue sortite in avanti, specie quando nell'ultima mezz'ora il tecnico lo sposta in mediana [in linea mediana, n.d.a.] e lui confeziona il cross per Hoffer, puntuale all'appuntamento col gol, 14' dopo il suo esordio (gazzetta.it, 17-8-09).

\subsection{L'ASPETTO SEMANTICO}

Come ogni lingua settoriale, la lingua del calcio ricorre alle collocazioni. Sobrero nota giustamente che, a differenza di quelle specialistiche, come la lingua della fisica o dell'informatica, le lingue settoriali ,[...] non dispongono di un lessico specifico vero e proprio - o meglio, dispongono di un lessico specifico molto ridotto - né di regole convenzionali particolari, ma attingono spesso alla lingua comune o ad altre lingue speciali, importandone parole, espressioni, metafore" ${ }^{24}$. Questa caratteristica è dovuta al fatto che „I messaggi delle lingue settoriali si diffondono in gran parte attraverso i mass media: la minore specializzazione del loro lessico, e i numerosi contatti con la lingua comune sono dovuti alla necessità di farsi capire da un'utenza molto ampia e indifferenziata" ${ }^{\prime 2}$.

Ciononostante la lingua del calcio, come si vedrà attraverso la nostra analisi, ha un lessico e delle regole specifici riguardanti il modo di formare i neologismi e le strutture testuali. Nel linguaggio giornalistico sportivo per definire l'azione di fare gol vengono adoperate diverse collocazioni: far rete, segnare / siglare una rete, baciare la rete, metter dentro, conseguire / marcare / fare / realizzare / firmare un gol (però si

${ }^{19}$ La Gazzetta dello Sport, 18-9-2003, p. 5.

${ }^{20}$ Figura consistente nel rovesciamento dell'ordine degli elementi nella frase; v. a proposito Moretti (1996, p. 669).

${ }^{21}$ La Gazzetta dello Sport, 18-9-2003, p. 3

${ }^{22}$ Figura sintattica di ripetizione che consiste nel collegare varie parole o frasi mediante la ripetizione di congiunzioni coordinative; cfr. Moretti (1996, p.674).

${ }^{23}$ Tuttosport, 18-9-2003, p. 5.

${ }^{24}$ Sobrero (a c. di), (1993, p. 239).

${ }^{25}$ Sobrero (a c. di), (1993, p. 239). 
dice trovare gol), andare in gol / a bersaglio. Altre collocazioni frequenti sono fare / adattare il turnover. Tra le collocazioni grammaticali troviamo marcare a uomo (sottoporre a marcamento stretto un attaccante avversario), marcare a zona (eseguire un marcamento in un certo settore del campo); assistente dell'allenatore viene definito allenatore in seconda, la punizione indiretta - calcio a due e la partita delle 20.30 si dice giocata in notturna. Tra gli altri modi di dire segnaliamo fare pressing / pressare, fare / effettuare un cross / un traversone, partire in contropiede. C'è pertanto una sfumatura tra centrare il poker e fare poker, dove nell'ultima espressione viene ommesso l'articolo. Si usa anche dire, quando ha luogo nei minuti finali di una partita un clamoroso salvataggio della porta, di salvare in extremis / allo scadere; e un giocatore svincolatosi da un contratto che lo legava ad una squadra viene detto liberato in extremis.

Osserviamo anche la fitta presenza di verbi con complemento oggetto: giocare unica punta (da attaccante avanzato), giocare titolare, promuovere qualcuno titolare, sponsorizzato Nike. Un giocatore può essere acquistato (non comprato, infatti si dice campagna acquisti o, eventualmente, campagna acquisti-cessioni) da una squadra, oppure ceduto (non prestato, semmai - può essere in prestito in un club); se invece accade il contrario, un calciatore è dichiarato incedibile / blindato. Quest'ultimo aggettivo si usa anche nei confronti di allenatore. Una squadra, quando perde, può crollare o cadere, mentre vincendo di gran lunga strapazza / travolge un'altra. La vincita stessa viene definita risultato utile. Del direttore di gara, oltre a arbitrare, si suol dire che fischia una partita. Come osserviamo sugli esempi soprastanti, la lingua del calcio fa un largo uso delle collocazioni.

\subsection{L'ASPETTO SINTATTICO}

I linguaggi settoriali presentano, oltre al lessico caratteristico, anche particolari soluzioni sintattiche. In primo luogo vogliamo sottolineare l'espansione del nome rispetto al verbo, dettata dalle ragioni di sinteticità. La forte nominalizzazione dei titoli viene adoperata allo scopo di cogliere l'attenzione del lettore potenziale e incuriosirlo dosandogli l'informazione a poco a poco, però mettendolo sempre prima davanti ad un fatto compiuto:

„Campioni del mondo" - in questo modo La Gazzetta dello Sport salutò nel 1982 la conquista del titolo mondiale dalla Nazionale Azzurra. Ricordiamo che il linguaggio dello stesso giornale ai tempi della dittatura fascista, al contrario, fu eccessivamente enfatico, le frasi - ridondanti, si sprecavano gli aggettivi e le vittorie venivano celebrate nel nome di Mussolini. Dopo la vittoria dei giocatori azzurri ai Mondiali di calcio nel 1938 i titoli della prima pagina del 20 giugno furono irruenti e grandiosi:

Apoteosi dello sport fascista nello stadio di Parigi / Gli azzurri splendenti di slancio e stile partono all'offensiva [...] e trionfano nella luce di un gioco magistrale. 
Come altro esempio possiamo citare „MONDIALEX / Col rientro di Diego, Del Piero torna a guidare l'attacco Juve / Il capitano ricomincia la caccia al gol e a Sud Africa 2010" (Ts, 13-8, p. 1) - titolo che costituisce una prova rilevante di come sia sintetico e nominale lo stile del linguaggio giornalistico che sotto alcuni aspetti richiama quello telegrafico. Infatti la frase suonerà enigmatica nelle orecchie dei profani del calcio e solo i calciofili sapranno che si tratta di una auspiccio che il capitano della squadra della Juventus, Alessandro Del Piero, inauguri bene la nuova stagione e conquisti così la convocazione nella Nazionale in vista dei Mondiali di Calcio. Un altro evento viene riportato da Tuttosport con altrettanta sinteticità: „Goduria Juve! / Amauri rimonta l'Inter, ko ai rigori, e con Iaquinta che stende il Milan" (Ts, 15-8-09, p. 1), od ancora: „Voglia di Maxi Juve (tit.) / I bianconeri sfidano Inter e Milan per sfatare il tabů del Tim Sondaggio con l'Atletico Madrid: nel mirino c'è Rodriguez" (Ts, 14-8-09, p.1).

Si noti però che l'uso ridotto o addirittura la mancanza dei verbi concerne spesso i titoli, il che si spiega, oltre ai motivi sopraelencati, con il bisogno di rendere per mezzo di parole lo svolgimento di un incontro calcistico. Infatti negli occhielli e nei sommari quel procedimento non viene adoperato, mentre nell'analisi di una partita a prevalere sono proprio le frasi nominali:

Promosso a pieni voti. La prima italiana di Diego, il brasiliano sulle cui spalle poggia la Juventus 2009/2010, è un successo completo. Gol benaugurante a San Siro contro il Milan nel Trofeo Berlusconi, prestazione da incorniciare e tifosi bianconeri che già si fregano le mani (gazzetta. it, 18-8-09).

Sempre riguardo alla sinteticità si osserva una quantità notevole di proposizioni subordinate temporali participiali: „Scardinata la resistenza dei padroni di casa, l'Inter soffre soltanto al 31'[...]”; ,Salutato da un boato della curva, Davids subentra a Tacchinardi nella ripresa". Questa scelta è dettata da ragioni di economia, il participio passato ha valore attivo nei verbi intransitivi (ad esempio scattato sulla destra, scattato sul filo di fuorigioco) e viene adoperato come elemento centrale di varie proposizioni dipendenti implicite (temporali, causali, concessive). È infatti più economico usare una forma implicita al posto della ipotattica esplicita. Quando viene analizzato l'andamento di una partita, per dare immediatezza ai fatti narrati si fa ricorso alle frasi semplici, sintetiche, sempre al presente indicativo (il cosiddetto presente storico), di solito prive di complementi:

Ripresa e la Juventus cambia volto. E volti. Si passa al 4-4-2 (sia pure tendente al 4-3-3), con linea di centrocampo formata da Salihamidzic, Marchisio, Melo e Iaquinta, mentre in attacco giostrano Giovinco e Amauri (Ts, 8-8-09, p. 2).

Grazie all'uso frequente del presente indicativo il lettore diventa facilmente partecipe della partita essendo in grado di seguire tutto quello che è successo in campo:

Sono i bianconeri ad andare subito vicino al gol. Bastano pochi minuti e l'azione, da manuale, è fatta tutta da tocchi di prima: Camoranesi innesca Giovinco, che serve Trezeguet, che fa spon- 
da per Del Piero il cui tiro è tuttavia preda di Diego Lopez. Il Villarreal dimostra comunque di non avere alcuna intenzione di stare a guardare e a rendersi pericoloso è proprio Rossi [...]: l'azzurro alza di poco sulla traversa, approfittando di una respinta non esattamente impeccabile di Manninger su tiro di Cazorla (Ts, 8-8-09, p. 2).

Di fatto dall'avvento della televisione (i Mondiali ' 54 furono i primi a essere trasmessi in Eurovisione) non è più sufficiente fare solo un semplice resoconto di una partita, la concorrenza del nuovo mezzo impone un cambiamento nel modo di servire il lettore. La cronaca dell'avvenimento non basta più, poiché chi legge ha già visto che cosa era accaduto in campo e vuole conoscerne sia i perché che i retroscena. Si tratta di andare oltre la notizia, occorre tirar fuori l'uomo che c'è dietro ogni campione. Il giornalista non è più chiamato a esprimere un parere illuminato né a rivelare agli sportivi le verità di cui è l'unico dipositario, deve far partecipare il lettore e coinvolgerlo emotivamente. Questo è il compito della stampa sportiva che segue il dispositivo di B. Lamy: ,per rendere il nostro discorso più efficace dobbiamo figurarlo, ossia dobbiamo dargli il carattere delle nostre passioni" ${ }^{26}$.

Oltre al presente storico altri tempi sono assai poco frequenti, mentre sono diffusi i verbi transitivi, come per esempio pressare qlcu, infilare qlcu (detto di un portiere a cui viene segnato un gol), dribblare qlcu, lanciare qlcu (farlo scattare). Altrettanto rilevante pare l'alta ricorrenza del participio presente - modo indefinito con un valore attivo („Amauri e Iaquinta devastanti” [Ts, 15-8-09, p. 2]), di avverbi in funzione di aggettivi (giornata no, momento no, rigore no) e di aggettivi in funzione avverbiale (,Moduli speculari: 4-3-1-2 sia per Leonardo che per Ferrara, ma le punte del Milan, per favorire gli inserimenti di Dinho, giocano più larghe di quelle bianconere [stasera Trezeguet e Iaquinta]”, gazzetta.it, 17-8-09; „Le squadre giocano alte, in pochi metri, in particolare per il Milan funziona bene la tattica del fuorigioco" [gazzetta.it, 17-8-09]; „Ali Cesare Prandelli quest'anno li ha provati tutti, e parliamo di moduli. Le tre punte, il rombo, il 4-4-2, varianti varie per poi arrivare a questo 4-2-3-1 nel quale i due esterni dei tre dietro a Gilardino stanno larghi e colpiscono, perché lo sanno fare" (gazzetta.it, 27-4-09); „Berlusconi ha anche spazzato via le voci sulla possibile cessione del Milan [«voi rappresentate una realtà che non esiste», dice ai cronisti] e sulla squadra vista ieri sera non risparmia consigli per Huntelaar [ «aspettiamo prima di dare un giudizio, aspettiamo che si inserisca, deve però stare molto più centrale, l'ho detto anche all'allenatore»]" (gazzetta.it, 18-8-09).

\section{NOTE CONCLUSIVE}

Come si è visto nel presente saggio, a sorpresa dei profani del pallone, quella del calcio è una vera e propria lingua settoriale. L'analisi della scrittura giornalistica di calcio, quindi, in base al corpus studiato, permette di constatare che si tratta di un set-

${ }^{26}$ Lamy (1968), cit. in Genette (1969, p. 199). 
tore intriso sia di tecnicismi collaterali che di quelli specifici. Come abbiamo notato, i tecnicismi - caratteristica rilevante del sottocodice pallonaro, costituiscono la parte del leone di una cronaca calcistica. Vale la pena riportare l'opinione di Bonomi (2002, p. 226) la quale nota che „La grande categoria degli articoli dedicati ad argomenti settoriali deve essere differenziata al suo interno fondamentalmente, mi pare, tra articoli di economia, di divulgazione tecnico-scientifica, di cultura, di spettacolo, di sport".

In conclusione si può affermare che ciò che è emerso dallo spoglio costituisce una rilevante prova dell'abbondanza metaforica del linguaggio delle cronache calcistiche. Dello stesso parere rimane la linguista $(2002$, p. 227) sostenendo che ,gli articoli sportivi presentano una struttura compositiva e soprattutto una caratterizzazione linguistica molto differenziate rispetto al resto del quotidiano, con una forte accentuazione della componente espressiva".

Ed infine, effettuata l'analisi dei materiali ed esaminati i molteplici aspetti del linguaggio calcistico, abbiamo avuto la conferma dell'esistenza di variegati tratti della lingua settoriale del calcio, da quelli stilistico-testuali a quelli semantici e sintattici.

\section{BIBLIOGRAFIA}

Adamo, G., \& Della Valle, V. (2005). Parole nuove. Un dizionario di neologismi dai giornali. Milano: Sperling \& Kupfer Editori.

Berruto, G. (1976). Sociolinguistica dell'italiano contemporaneo. Bologna: Il Mulino.

Bonomi, I. (2002). Il linguaggio giornalistico. Firenze: Franco Cesati Editore.

De Mauro, T. (2000). Il dizionario della linugua italiana per il terzo millennio. Torino: Paravia.

Devoto, G. (1939). Le lingue speciali: le cronache del calcio. Lingua Nostra, I, 17-21.

Genette, G. (1969). Figure. Retorica e strutturalismo. Torino: Einaudi.

Lamy, B. (1688, ed.1968). La réthorique ou l'art de parle. In G. Genette. Figure. Retorica e strutturalismo. Torino: Einaudi.

Moretti, G.B. (1996). L'italiano come prima o seconda lingua nelle sue varietà scritte e parlate. Grammatica descrittiva di riferimento con prontuario di verbi regolari e irregolari con indicazioni di analisi testuale con note di retorica e stilistica. (Vol. I). Perugia: Edizioni Guerra.

Roidi, V. (2001). La Fabbrica delle Notizie. Bari: Laterza.

Serianni, L. (2003). Italiani scritti. Bologna: Il Mulino.

Sobrero, A.A. (1993). Introduzione all'italiano contemporaneo. La variazione e gli usi. Roma: Lingue speciali.

Zingarelli, N. (2004). Il Nuovo Zingarelli Minore. Vocabolario della lingua italiana. Bologna: Zanichelli.

www.audipress.it. (n.d.).

www.gazzetta.it. (n.d.).

www.repubblica.it. (n.d.). 